\title{
Repercussões para a gestão oriundos da judicialização de medicamentos: uma
}

\section{revisão integrativa}

\author{
Management repercussions from the judicialization of medicines: an integrative review \\ Repercusiones para la gestión de la judicialización de medicamentos: una revisión integrativa
}

Recebido: 05/02/2021 | Revisado: 08/02/2021 | Aceito: 09/02/2021 | Publicado: 17/02/2021

\author{
Cinara Rejane Viana Oliveira \\ ORCID: https://orcid.org/0000-0002-4835-2481 \\ Universidade do Estado da Bahia, Brasil \\ E-mail: cinara-rejane@hotmail.com \\ Emily Maria Torres de Magalhães Borges \\ ORCID: https://orcid.org/0000-0002-5267-2402 \\ Universidade do Estado da Bahia, Brasil \\ E-mail: mily_borges@ hotmail.com \\ Sérgio Donha Yarid \\ ORCID: https://orcid.org/0000-0003-0232-4212 \\ Universidade Estadual do Sudoeste da Bahia, Brasil \\ E-mail: yarid@uesb.edu.br
}

\begin{abstract}
Resumo
Mesmo com a implantação da Política Nacional de Medicamentos (PNM), o acesso aos medicamentos ainda é limitado para grande parte da população brasileira sendo como única alternativa recorrer a judicialização. Os gastos com os medicamentos judicializados não são previstos nos planos anuais de saúde e nas Leis Orçamentárias Anuais, o que acarreta muitas vezes em despesas não programadas. O presente estudo trata-se de uma revisão integrativa, com característica quantitativa, realizada no período de fevereiro a agosto de 2020. A coleta de dados foi realizada utilizando-se as bases de dados: National Center for Biotechnology Information (PUBMED), Scientific Electronic Library Online (SCIELO), Literatura Latino-americana e do Caribe em Ciências da Saúde (LILACS) e sites confiáveis. Observou-se que a judicialização de medicamentos vem aumentando progressivamente em todo país, exigindo que o gestor subsidie o processo com as informações que dispor para uma decisão mais adequada. Também, outras medidas como a inserção de uma equipe multiprofissional auxiliando os juízes no julgamento das ações judiciais; maior conscientização dos prescritores sobre a importância de prescrever medicamentos existentes na Relação de Medicamentos Essenciais e um maior investimento em Pesquisa e desenvolvimento, são alguns pontos relevantes que podem ajudar a reduzir e racionalizar a judicialização de medicamentos.
\end{abstract}

Palavras-chave: Judicialização; Medicamentos; Assistência farmacêutica.

\begin{abstract}
Even with the implementation of the National Medicines Policy (PNM), access to medicines is still limited for a large part of the brazilian population, leading to judicialization as an alternative. As judicialized medicines are not foreseen in the annual health plans and the annual budget laws, this situation often results in unscheduled expenses. This study is an integrative review, with a quantitative characteristic, carried out from February to August 2020. Data collection was performed using the following databases: National Center for Biotechnology Information (PUBMED), Scientific Electronic Library Online (SCIELO), Literatura Latino-americana e do Caribe em Ciências da Saúde (LILACS) and trusted sites. It was observed that the judicialization of medicines has been progressively increasing across the country, requiring the manager to subsidize the process with the information he has at his disposal for a more appropriate decision. Also, other measures such as the insertion of a multiprofessional team assisting the judges in the judgment of lawsuits; greater awareness among prescribers about the importance of prescribing existing drugs in the Relação de Medicamentos Essenciais and a greater investment in Research and Development are some relevant points that can help to reduce and rationalize the judicialization of medicines.
\end{abstract}

Keywords: Judicialization; Medicines; Pharmaceutical care.

\section{Resumen}

A pesar de la implantación de la Política Nacional de Medicamentos (PNM), el acceso a los medicamentos sigue siendo limitado para gran parte de la población brasileña siendo como única alternativa recurrir a la judicialización. Los gastos en medicamentos judicializados no están previstos en los planes anuales de salud y en las Leyes Presupuestarias Anuales, lo que a menudo implica gastos no programados. El presente estudio es una revisión integral, con carácter cuantitativo, realizada en el período comprendido entre febrero y agosto de 2020. La 
recopilación de datos se realizó utilizando las bases de datos: National Center for Biotechnology Information (PUBMED), Scientific Electronic Library Online (SCIELO), Literatura Latino-americana y del Caribe en Ciencias de la Salud (LILACS) y sitios confiables. Se ha observado que la judicialización de medicamentos va en aumento progresivamente en todo el país, exigiendo que el gestor subvencione el proceso con las informaciones que disponga para una decisión más adecuada. También, otras medidas como la inserción de un equipo multiprofesional ayudando a los jueces en el juicio de las acciones judiciales; mayor concienciación de los prescriptores sobre la importancia de prescribir medicamentos existentes en la Relación de Medicamentos Esenciales y una mayor inversión en Investigación y desarrollo, son algunos puntos relevantes que pueden ayudar a reducir y racionalizar la judicialización de medicamentos.

Palabras clave: Judicialización; Medicamentos; Asistencia farmacéutica.

\section{Introdução}

Um dos grandes objetivos definidos pelo Sistema Único de Saúde (SUS), através da Lei 8.080/90, pauta-se na promoção e proteção à saúde de toda população, tendo o acesso aos medicamentos como um importante fator no alcance desse objetivo (Brasil, 1990).

Em 1998, através da portaria n 3.916 , foi a publicada a Política Nacional de Medicamentos (PNM), cuja finalidade principal é "garantir a segurança, eficácia e qualidade dos medicamentos, a promoção do uso racional e o acesso da população aos medicamentos considerados essenciais" (Brasil, 1998). Assim, uma das diretrizes prioritárias da PNM consistiu na reformulação da Assistência Farmacêutica (AF), fundamentada na descentralização da gestão, na promoção do uso racional de medicamentos, na otimização do sistema de distribuição e no desenvolvimento de iniciativas que possibilitem a redução dos preços dos produtos (Brasil, 1998).

Com a criação da Política Nacional de Assistência Farmacêutica (PNAF) em 2004, como parte integrante da PNM, a Assistência Farmacêutica (AF) foi definida como um "conjunto de ações voltadas à promoção, proteção e recuperação da saúde, tendo o medicamento como insumo essencial, visando o acesso e ao seu uso racional” (Brasil, 2004).

Desde épocas passadas perpassando a atualidade, um dos grandes desafios para a gestão da AF no SUS constitui-se em estabelecer um processo sustentável de garantia do financiamento e, consequentemente, do acesso aos medicamentos frente aos escassos recursos disponíveis e os elevados custos da assistência à saúde.

Com a publicação da Portaria no 204/GM de 29 de janeiro de 2007 regulamentou-se o financiamento e a transferência em forma de blocos de financiamento dos recursos federais para as ações e os serviços de saúde. O bloco da Assistência Farmacêutica ficou dividido em três Componentes: o Componente Básico, destinado ao custeio de ações da AF relacionadas às doenças e aos agravos mais prevalentes; o Componente Estratégico, destinado ao custeio de ações da AF relacionadas a doenças epidêmicas e endêmicas, e o Componente Especializado da Assistência Farmacêutica (CEAF) como uma estratégia de acesso a medicamentos no âmbito do SUS, em nível ambulatorial, por meio de linhas de cuidado definidas em Protocolos Clínicas e Diretrizes Terapêuticas (PCDT), publicadas pelo Ministério da Saúde [MS] (Brasil, 2013).

Porém, a dificuldade de acesso à farmacoterapia através dos componentes da AF tem culminado no incremento de vias alternativas de acesso aos medicamentos desses componentes, como a via judicial. Os gastos com a judicialização de medicamentos não constam nos planos anuais de saúde e nas Leis Orçamentárias Anuais. O custeio das ações judiciais é garantido através da utilização de recursos dos componentes existentes, resultando no aumento exponencial de gastos com a Assistência Farmacêutica.

A judicialização da saúde influencia diretamente na execução das políticas no âmbito do Sistema Único de Saúde (SUS), uma vez que pode gerar gastos elevados e não programados. Como exemplo, no Estado da Bahia, no ano de 2015, foram impetradas 774 ações judiciais contra o Estado (Oliveira et al.,2016).

Apesar das repercussões negativas da judicialização de medicamentos, é sabido que o Estado tem por obrigação garantir tratamento para o cidadão acometido por uma doença no qual o medicamento possui preço elevado, não está 
disponível nas outras instâncias da AF ou caso não possua condições financeiras para comprar mesmo com baixo preço (Carvalho, 2019).

Dentro desse contexto, o presente trabalho, que terá o supracitado objeto de pesquisa, identificará questões inerentes a judicialização de medicamentos, destacando pontos favoráveis e desfavoráveis dentro do contexto.

\section{Metodologia}

Trata-se de uma revisão integrativa com característica quantitativa, realizada no período de fevereiro a agosto de 2020.

Para descrever a metodologia utilizou-se como referência Mendes, Silveira e Galvão (2008), que define como etapas: identificação do tema e seleção da hipótese ou questão de pesquisa para a elaboração da revisão integrativa; estabelecimento de critérios para inclusão e exclusão de estudos/ amostragem ou busca na literatura; definição das informações a serem extraídas dos estudos selecionados/ categorização dos estudos; avaliação dos estudos incluídos na revisão integrativa; interpretação dos resultados; apresentação da revisão/ síntese do conhecimento.

Foi levantada a seguinte questão norteadora para concretização da pesquisa: "quais são as consequências que a literatura aborda diante a judicialização de medicamentos para a população e para o orçamento público"?

A coleta de dados foi realizada utilizando-se as bases de dados: National Center for Biotechnology Information (PUBMED), Scientific Electronic Library Online (SCIELO) e a Literatura Latino-americana e do Caribe em Ciências da Saúde (LILACS).

Como critérios de inclusão, utilizaram-se artigos originais publicados entre os anos de 2015 e 2020, disponíveis na íntegra, que abordaram a temática judicialização/demandas judiciais envolvendo o acesso a medicamentos.

Os operadores boreanos "AND" e "AND NOT" foram utilizados para melhor selecionar os artigos diante a devido a grande quantidade de artigos disponíveis que abordavam do assunto.

Figura 1. Etapas para a seleção e escolha dos artigos utilizados no estudo.

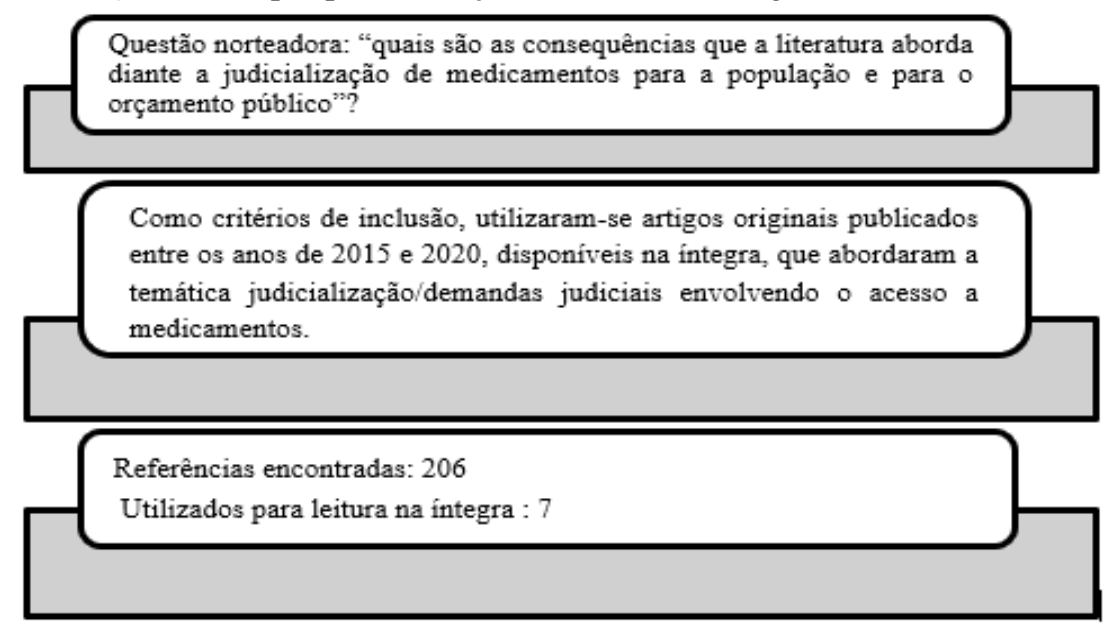

Fonte: Autores.

\section{Resultados}

Foram selecionados 7 trabalhos, após uma leitura pormenorizada. Os critérios utilizados na escolha deram-se através da similaridade dos conteúdos, dando prioridade os que destacavam sobre os gastos com a judiciadização de medicamentos, consequências positivas e negativas da judicialização, lacunas entre direito ao acesso de medicamentos e suas repercussões na 
saúde pública além de possíveis soluções para problemática da judicialização dos medicamentos de alto custo no Brasil. Os artigos escolhidos estão listados abaixo no Quadro 1, também foram utilizados outros trabalhos para embasamento do estudo.

Quadro 1. Artigos encontrados nas bases de dados que retratam sobre as repercussões da judicialização de medicamentos no Brasil.

\begin{tabular}{|c|c|c|c|}
\hline Título do Artigo & Autores & Resultados & Considerações finais \\
\hline $\begin{array}{l}\text { 1. A judicialização e } \\
\text { seu papel na } \\
\text { efetivação do direito á } \\
\text { saúde pública }\end{array}$ & $\begin{array}{l}\text { Fabiana Diniz } \\
\text { Lopes, Tatiana } \\
\text { Ribeiro de Campos } \\
\text { Mello }\end{array}$ & $\begin{array}{l}\text { Nos municípios da Região Leste Metropolitana de } \\
\text { São Paulo existe uma tendência crescente do } \\
\text { aumento da judicialização da saúde, assim como no } \\
\text { país como um todo. }\end{array}$ & $\begin{array}{l}\text { São necessários mais } \\
\text { estudos sobre a judicialização da } \\
\text { saúde para que seja possível } \\
\text { preservar o direito fundamental à } \\
\text { saúde do indivíduo. }\end{array}$ \\
\hline $\begin{array}{l}\text { 2. A Judicialização de } \\
\text { medicamentos no } \\
\text { estado da Bahia: os } \\
\text { números no período } \\
\text { de } 2014 \text { a } 2017\end{array}$ & $\begin{array}{l}\text { Poliana Brito } \\
\text { Barbosa, Simone } \\
\text { Cerqueira Machado } \\
\text { Alves }\end{array}$ & $\begin{array}{l}\text { É inegável e notória a tendência de crescimento das } \\
\text { demandas, estando de acordo com a literatura } \\
\text { existente e com os dados do próprio CNJ na área da } \\
\text { saúde. O orçamento total para medicamentos em } \\
2017 \text { na Bahia foi de } \mathrm{R} \$ 175.331,407,00 \text {, os } \\
\text { pacientes com decisões judiciais fizeram jus, per } \\
\text { capita, a } \mathrm{R} \$ 29.221,90 \text {, enquanto que, para a } \\
\text { população em geral, ficariam cerca de } \mathrm{R} \$ 7,01\end{array}$ & $\begin{array}{l}\text { O estudo apresenta o impacto no } \\
\text { estado da Bahia, demonstrando que } \\
\text { também existem falhas na política } \\
\text { pública, podendo ajudar gestores na } \\
\text { tomada de decisões, além de servir } \\
\text { de motivador para pesquisas } \\
\text { futuras. }\end{array}$ \\
\hline $\begin{array}{l}\text { 3. A judicialização } \\
\text { dos medicamentos de } \\
\text { alto custo no Brasil e } \\
\text { a teoria dos custos dos } \\
\text { direitos }\end{array}$ & $\begin{array}{l}\text { Adriano Rodrigo } \\
\text { Reis, Leandro } \\
\text { Correa de Oliveira. }\end{array}$ & $\begin{array}{l}\text { As políticas públicas de dispensação de } \\
\text { medicamentos desenvolvidas pelos demais órgãos } \\
\text { da administração pública, demonstra que as } \\
\text { questões relacionadas ao custo dos direitos são } \\
\text { relegadas a um segundo plano, como se qualquer } \\
\text { tecnologia existente no mercado mundial de } \\
\text { medicamentos fosse passível de ser custada pelo } \\
\text { estado brasileiro. }\end{array}$ & $\begin{array}{l}\text { A discussão sobre os parâmetros e } \\
\text { critérios a serem observados pelos } \\
\text { magistrados em tais demandas, } \\
\text { verifica-se que não há } \\
\text { aprofundamento nas discussões } \\
\text { sobre questões envolvendo o valor a } \\
\text { ser dispendido pelo estado e suas } \\
\text { consequências para o orçamento } \\
\text { público. }\end{array}$ \\
\hline $\begin{array}{l}\text { 4. Características da } \\
\text { judicialização } \\
\text { acesso do } \\
\text { medicamentos r no } \\
\text { Brasil: uma revisão } \\
\text { sistemática }\end{array}$ & $\begin{array}{l}\text { Izamara Damasceno } \\
\text { Catanheide, Erick } \\
\text { Soares Lisboa,Luis } \\
\text { Eugenio Portela } \\
\text { Fernandes de Souza }\end{array}$ & $\begin{array}{l}\text { Os } 53 \text { estudos empíricos revisados revelam tanto o } \\
\text { que se sabe sobre o fenômeno atual da } \\
\text { judicialização do acesso a medicamentos no Brasil, } \\
\text { quanto os aspectos pouco esclarecidos e as lacunas } \\
\text { do conhecimento. }\end{array}$ & $\begin{array}{l}\text { Esta revisão identifica: os desfechos } \\
\text { da utilização do medicamento } \\
\text { demandado judicialmente, o grau de } \\
\text { adesão dos prescritores às listas } \\
\text { oficiais e a participação da indústria } \\
\text { e das associações de portadores de } \\
\text { doenças nesse processo. }\end{array}$ \\
\hline $\begin{array}{l}\text { 5. Judicialização da } \\
\text { saúde: a dignidade da } \\
\text { pessoa humana e a } \\
\text { atuação do Supremo } \\
\text { Tribunal Federal no } \\
\text { caso dos } \\
\text { medicamentos de } \\
\text { alto custo* }\end{array}$ & $\begin{array}{l}\text { Fabricio Veiga } \\
\text { Costa } \\
\text { Ivan Dias da Motta } \\
\text { Dalvaney Aparecida } \\
\text { de Araújo }\end{array}$ & $\begin{array}{l}\text { Diversos casos são submetidos à apreciação judicial } \\
\text { para que se cumpra o mister constitucional de } \\
\text { prestação universalizada dos serviços de saúde. } \\
\text { Embora se trate de uma verdadeira missão } \\
\text { constitucional delegada indiretamente aos juízes e } \\
\text { tribunais, a judicialização apresenta graves } \\
\text { problemas advindas da falta de critérios e } \\
\text { voluntarismos diversos de decisões irrazoadas }\end{array}$ & $\begin{array}{l}\text { O Estado tem o dever de fornecer } \\
\text { medicamentos de alto custo, } \\
\text { ressaltando-se que ao judiciário } \\
\text { caberá a atribuição de interpretar tal } \\
\text { direito de forma sistemática, } \\
\text { constitucionalizada, democrática, } \\
\text { integrativa, inclusiva e isonômica, } \\
\text { garantindo-se a todos os cidadãos, } \\
\text { indistintamente. }\end{array}$ \\
\hline $\begin{array}{l}\text { 6. Offset uma possível } \\
\text { solução para } \\
\text { problemática da } \\
\text { judicialização dos } \\
\text { medicamentos de alto } \\
\text { custo no } \\
\text { Brasil }\end{array}$ & $\begin{array}{l}\text { Débora } \\
\text { Carvalho }\end{array}$ & $\begin{array}{l}\text { O Brasil precisa, urgentemente, acelerar sua forma } \\
\text { de atuação no âmbito da pesquisa, desenvolvimento } \\
\text { e inovação. É preciso aprender com os países } \\
\text { desenvolvidos, e de alta competitividade, a fim de } \\
\text { possibilitar que o Brasil consiga realizar uma } \\
\text { operação de catching up e em um período de longo } \\
\text { prazo se estabeleça dentre o ranking dos países } \\
\text { mais inovadores }\end{array}$ & $\begin{array}{l}\text { O produto tecnológico criado tem a } \\
\text { intenção dedivulgar o que é o offset } \\
\text { e suas formas de modelagem, } \\
\text { fazendo com que cada vez mais as } \\
\text { instituições públicas tenham } \\
\text { conhecimento desse ativo e propor } \\
\text { o uso do offset do tipo comercial } \\
\text { em busca de uma negociação } \\
\text { countertrade como tentativa de } \\
\text { solucionar, a longo prazo, a, } \\
\text { problematização do gasto } \\
\text { exorbitante com medicamentos de } \\
\text { alto custo. }\end{array}$ \\
\hline
\end{tabular}




\begin{tabular}{|c|c|c|c|}
\hline $\begin{array}{lr}\text { 7. Medicamentos } & \text { de } \\
\text { alto custo: } & \text { a } \\
\text { judicialização e } & \text { o } \\
\text { papel do Estado. } & \end{array}$ & $\begin{array}{l}\text { Suzana Maria } \\
\text { Fernandes } \\
\text { Mendonça }\end{array}$ & $\begin{array}{l}\text { A garantia constitucional à saúde em sua forma } \\
\text { plena caracteriza-se como direito de todos e dever } \\
\text { do Estado. É um bem que está sob tutela do Poder } \\
\text { Público, que deve efetivá-lo de maneira a suprir as } \\
\text { necessidades de todos, ainda que apresentem } \\
\text { carências diversas e pesadas, sob a perspectiva } \\
\text { orçamentária. }\end{array}$ & $\begin{array}{l}\text { Os medicamentos de alto custo, } \\
\text { assim, são devidos pelo Poder } \\
\text { Público desde que comprovada a } \\
\text { necessidade do paciente e a sua } \\
\text { adequação ao respectivo } \\
\text { tratamento. Mostra-se inviável, } \\
\text { portanto, a abstenção do Estado no } \\
\text { atendimento mesmo que de apenas } \\
\text { um indivíduo. }\end{array}$ \\
\hline
\end{tabular}

Fonte: Autores.

\section{Discussão}

O financiamento para o custeio do SUS foi definido na Portaria n 204/GM, de 29 de janeiro de 2007. Nessa linha, regulamentou-se o financiamento e a transferência dos recursos federais para as ações e os serviços de saúde na forma de blocos de financiamento, incluso o da AF. Portanto, ficou definido que seria competência de todos os entes federativos (União, Estados, Distrito Federal e Municípios) promoverem a estrutura da assistência farmacêutica e garantir o acesso da população aos medicamentos e o seu uso racional, em conjunto com as demais esferas de governo (Brasil, 2007).

Apesar da implementação das políticas públicas no Brasil ocorridas na década de 1990, a disponibilização de medicamentos de forma contínua e em quantidade adequada às necessidades da população ainda é um desafio a ser superado.

$\mathrm{O}$ acesso aos medicamentos pelo sistema público de saúde é principalmente para a parcela população mais carente a única via de acesso aos medicamentos. E infelizmente, apesar da relevância do SUS na provisão de fármacos, em um estudo realizado por Boing e colaboradores em 2013, demonstrou que apenas 45,3\% das pessoas que têm medicamentos prescritos em consultas no sistema público os obtêm integralmente no próprio SUS.

A limitação no acesso aos medicamentos está relacionada a várias questões, a exemplo da piora do estado de saúde, do maior uso de terapias adicionais, aumento no número de retornos aos serviços de saúde e gastos adicionais nos tratamentos (Arrais et al., 2005).

A falta de efetividade das políticas públicas acaba gerando a judicialização. Os gastos com medicamentos decorrentes desse fenômeno jurídico não constam nos planos anuais de saúde e nas Leis Orçamentárias Anuais, com o custeio das respectivas ações sendo garantido através da utilização de recursos dos componentes existentes, resultando no aumento exponencial de gastos com a AF.

A tendência da judicialização é causar impactos na política de saúde principalmente dos municípios que, por sua vez, possuem menor capacidade para lidar com os custos e a imprevisibilidade advinda dos gastos em saúde ordenados judicialmente.

A judicialização deve ser realizada com cautela para estar evitando gastos elevados e desnecessários. Portanto, devese analisar a questão de maneira mais ampla, observando não só o medicamento e o direito do paciente ao acesso do tratamento, mas também a real necessidade de utilização de determinada tecnologia e a sua adequação ao tratamento, bem como a possibilidade de custeio do Estado (Mendonça, 2015).

Devido aos grandes impactos causados pela judicialização, muitos pontos devem ser considerados antes de judicializar um medicamento, sendo que esse questionamento se agrava quando existem tratamentos alternativos igualmente eficazes aos solicitados (Mendonça, 2015).

Em um trabalho realizado por Provin e colaboradores em 2013, demonstrou que a maior parcela populacional que solicitava medicamentos judicialmente eram pacientes do sistema privado de saúde e que tinham boas condições e que na maioria das vezes no sistema público de saúde obtinha o medicamento solicitado ou alguma alternativa similar. Assim, chegase as seguintes conclusões: a parte mais carente da população em sua maioria desconhece o processo da judicialização ou não 
possuem condições financeiras e instruções de como adquirir medicamentos via judicial e por outro lado a população com melhores condições financeiras desconhecem os componentes do SUS ou são instruídos a adquirir o medicamento sem mesma orientação quanto a outras alternativas.

Considerando a disponibilidade de alternativas terapêuticas mostra que a gestão do SUS não é totalmente omissa na efetivação do acesso a medicamentos (Machado et al., 2011).

De acordo com uma revisão sistemática de literatura realizada por Catanheide e colaboradores em 2016, ao comparar resultados de artigos percebeu-se que a judicialização de medicamentos pertencentes aos componentes da assistência farmacêutica do SUS estão presentes de modo significativo, porém, há um predomínio de medicamentos que não fazem parte das listas oficiais.

A Assistência farmacêutica é a área da saúde que mais sofre transformações devido às constantes inovações tecnológicas (Souza, 2016). Portanto, a judicialização de medicamentos pode ser influenciada pela indústria farmacêutica, sendo necessária uma maior cautela antes de deferir as ações judiciais.

Como verificado no trabalho elaborado por Correa e colaboradores em 2019, o Supremo Tribunal Federal (STF) adota uma perspectiva substancialista do direito, posicionando-se a favor da Assistência Farmacêutica em detrimento do interesse econômico-financeiro do Estado, assim suas decisões defendem que questões de ordem orçamentária não podem interferir na efetivação do direito a saúde em sua matriz individual de atendimento. O STF traz como argumentação reconhecer os limites recursais do Estado de ordem financeiro-orçamentária, porém não abre mão da garantia de entrega do medicamento ao seu postulante individualmente.

Por outro lado, Costa e colaboradores aborda uma controvérsia. Ressalta que o STF tem utilizado vários critérios interpretativos para dificultar o exercício do direito fundamental da saúde como, por exemplo: Teoria da reserva do possível; finitude dos recursos públicos; prevalência do direito coletivo frente ao individual; obrigatoriedade de comprovação pelo cidadão de que ele e sua família não dispõem de condições financeiras para arcar com o pagamento do medicamento e tratamento médico; obrigatoriedade de inclusão do medicamento no rol do Sistema Único de Saúde; obrigatoriedade de aprovação da comercialização de medicamento pela ANVISA, essas entre muitas outras justificativas são contra os pedidos de fornecimento de medicamentos ao cidadão.

Solitariamente os ministros do STF criam seus próprios critérios que para julgamento das demandas referentes à saúde assumindo um papel de legislador e consequentemente limitando o acesso a saúde. O entendimento de defesa da unilateralidade e autocracia dos julgadores, correlacionando com solipsismo conclui-se que a única finalidade é certamente limitar o acesso a saúde aos cidadãos (Costa et al., 2017).

Na Bahia em 2017 os pacientes com decisões judiciais fizeram jus, per capita, de R\$29.221,90, já a verba total utilizada na compra de medicamentos foi de $\mathrm{R} \$ 175.331,407,00$. Retratando isso em porcentagem populacional, em 2017 de acordo com o Censo do IBGE de julho de 2017, a Bahia tinha cerca de 15,28 milhões de habitantes, assim para a população geral restou cerca de $\mathrm{R} \$ 7,01$, portanto menos de $1 \%$ da população ficou com 38,9\% de todo o orçamento para medicamentos, enquanto $99 \%$ foi dividido entre os $61,1 \%$ restantes (Barbosa \&Alves, 2019).

Em outras regiões do Brasil, como a exemplo da Região Leste metropolitana de São Paulo constata-se que existe uma tendência a favor do crescimento da judicialização da saúde o que é constatado para todo o país. Essa tendência pode ser justificada em falhas nas três esferas do governo e também pode estar refletindo interesses de grupos empresariais, principalmente da indústria farmacêutica visando reserva e aumento de mercado (Lopes \& Mello, 2018).

Diante de situações como essas cabe ao gestor utilizar seu poder discricionário para poder cumprir o que é determinado pelo Judiciário. Pois o definidor para o gestor é que a incorporação e uso da tecnologia tenham eficácia e sejam eficientes a partir de comprovações cientificas como por exemplos na medicina baseada em evidencias e que caibam no 
orçamento. Assim, cabe também a gestão fazer escolhas alocativas assertivas, para que se possa primar pela eficiência, em fazer o melhor com menos recursos.

Em outro ponto, partindo-se da percepção de que o Judiciário não domina conhecimentos específicos para julgar ações destinadas a medicamentos, é indiscutível a importância da presença de profissionais habilitados, a exemplo dos farmacêuticos, auxiliando-os em seus julgamentos.

Ao considerar que o juiz é um profissional extremamente capacitado, mas seu conhecimento muitas vezes se limita às praticas jurídicas, o trabalho multidisciplinar deve ser estimulado. A parceria principalmente entre juízes e farmacêuticos pode propiciar economia para os cofres públicos, além de combater a expansão desenfreada e sem critérios da judicialização.

Entre as diversas profissões da área da saúde, o farmacêutico destaca, pois obtém conhecimento específico sobre os medicamentos, em especial seus princípios ativos e as relações de medicamentos disponíveis no SUS, podendo assim estar fazendo intercambialidade entre medicamentos caso exista essa possibilidade, o que acaba diminuindo os valores dos medicamentos e reduzindo gastos públicos. Assim, cabe a ele orientar o juiz nesse sentido e dar base científica para que o juiz embase sua decisão e denegue, se necessário, o pedido. Isso também impede a expansão de lobbies farmacêuticos que buscam aumentar a venda de certos medicamentos que muitas das vezes possuem a mesma ação terapêutica dos medicamentos existentes nas listas do SUS (Silva, 2013).

Outra forma de diminuir a judicialização de medicamentos seria os prescritores utilizarem a lista Nacional de medicamentos essenciais (RENAME) em suas prescrições. A lista de medicamentos essenciais é um instrumento fundamental para organizar a Assistência Farmacêutica e para se garantir o acesso aos medicamentos atendendo as necessidades da população, portanto, a utilização da RENAME possibilita a aquisição padronizada dos medicamentos, com preços acessíveis, favorecendo o consumidor em geral e os gestores do Sistema, garantindo assim o uso seguro e racional de medicamentos (Margarinos et al., 2014).

Ao aprofundar em outras questões que interferem diretamente no aumento da judicialização, em especial de medicamentos caros, destaca-se a escassez de investimento em Pesquisa e Desenvolvimento (P\&D) no Brasil. A indústria farmacêutica ainda é concentrada na produção de genéricos não tendo incentivo para o estudo e desenvolvimento de novas drogas o que deixa o Brasil apenas como uma central de produção. Apesar da indústria farmacêutica brasileira constar na Pesquisa e Inovação (PINTEC) como exemplo de crescimento e transformação, certamente se houvesse maior investimento em P\&D seu potencial seria melhor aproveitado o que traria lucros para própria população e Estado (Carvalho, 2019).

Em suma, considerando que o Brasil é um país com mais de 210 milhões de habitantes, com grande área territorial e um Sistema Único de Saúde que abrange todos os brasileiros, a eficiência dos gastos em saúde é fundamental para que todos sejam assistidos, principalmente em um período de atual crise econômica.

\section{Conclusão}

Constatou-se pelo exposto que a judicialização de medicamentos repercute a discussões inacabáveis, pois a todo cidadão brasileiro é garantido o direito a saúde sendo que, por outro lado, o país não está preparado financeiramente para garantir esse direito igualmente para todos. Portanto, a inserção de uma equipe multiprofissional no julgamento das ações judiciais auxiliando os juízes, maior conscientização dos prescritores sobre a importância de prescrever medicamentos existentes na RENAME e também maior investimento em P\&D são alguns dos passos para começar a reduzir e racionalizar a judicialização de medicamentos.

A judicialização de medicamentos realizada de maneira desenfreada gera danos que envolvem todo o equilíbrio orçamentário do país podendo causar danos irreversíveis na economia. Portanto, a judicialização tenta sanar falhas no sistema 
de saúde, porém quando utilizada de forma irregular inverte a lógica das políticas públicas e, consequentemente, os princípios do SUS.

A solicitação recorrente por via judicial de medicamentos que não são incorporados no SUS remete a uma discussão sobre a abrangência e a competência técnica envolvidas na avaliação destes pleitos. Cabendo, portanto, uma melhor avaliação para a necessidade de incorporação de novas tecnologias farmacêuticas, da atualização sistemática das listas de medicamentos diante das inovações existentes, sua divulgação e também uma equipe multidisciplinar a fim de sanar dúvidas e despesas desnecessárias.

Em suma, com esse estudo foi possível analisar diferentes questões que interferem na judicialização de medicamentos, instigando assim a realização de novos estudos que demostrem qual a real situação do Brasil diante dos medicamentos judicializados e também trabalhos que visem novas alternativas que favoreçam a diminuição de custos pelo estado e ao mesmo tempo não deixe os cidadãos desassistidos.

\section{Referências}

Arrais, P. S. D., Brito, L. L., Barreto, M. L., \& Coelho, H. L. L. (2005). Prevalência e fatores determinantes do consumo de medicamentos no Município de Fortaleza, Ceará, Brasil. Cadernos de Saúde Pública, 21, 1737-1746.

Barbosa, P. B., \& Alves, S. C. M. (2019). A judicialização de medicamentos no estado da Bahia: os números no período de 2014 a 2017. Cadernos IberoAmericanos De Direito Sanitário, 8(4), 45-65.

Boing, A. C., Bertoldi, A. D., Boing, A. F., Bastos, J. L., \& Peres, K. G. (2013). Access to medicines in the public sector: analysis of users of the Brazilian Unified National Health System. Cadernos de saude publica, 29(4), 691-701.

Brasil (1999). Lei n ${ }^{\circ}$ 9.787, de 10 de fevereiro de 1999. Altera a Lei no 6.360, de 23 de setembro de 1976. Dispõe sobre a vigilância sanitária, estabelece o medicamento genérico, dispõe sobre a utilização de nomes genéricos em produtos farmacêuticos e dá outras providências. Diário Oficial da União. Brasília, DF, 10 de fev. 1999.

Brasil (1990). Lei $n^{\circ}$ 8.080, de 19 de setembro de 1990. Dispõe sobre as condições para a promoção, proteção e recuperação da saúde, a organização e o funcionamento dos serviços correspondentes e da outras providências. Diário Oficial da União. Brasília, DF, 19 set. 1990.

Brasil (1999). Lei no 9.782, de 26 de janeiro de 1999. Define o Sistema Nacional de Vigilância Sanitária, cria a Agência Nacional de Vigilância Sanitária, e dá outras providências. Diário Oficial da União. Brasília, DF, 26 de jan. 1999.

Brasil (2006). Ministério da Saúde. Secretaria de Ciência, Tecnologia e Insumos Estratégicos. Departamento de Assistência Farmacêutica e Insumos Estratégicos. Assistência farmacêutica na atenção básica: instruções técnicas para sua organização. 2.ed. Brasília, DF. 2006.

Brasil (2012). Ministério da Saúde. Portaria n ${ }^{\circ}$ 599, de 26 de junho de 2012. Aprova as Diretrizes Diagnósticas e Terapêuticas do Tumor Cerebral no Adulto. Diário Oficial da União. Brasília, DF, 26 jun. 2012.

Brasil (1998). Ministério da Saúde. Portaria no 3.916, de 30 de outubro de 1998. Dispõe sobre a Política Nacional de Medicamentos. Diário Oficial da União. Brasília, DF, 30 out. 1998.

Brasil (2004). Ministério da Saúde. Resolução n o 338, de 6 de maio de 2004. Aprova a Política Nacional de Assistência Farmacêutica. Diário Oficial da União. Brasília, DF, 6 de maio. 2004.

Brasil (2007). Ministério da Saúde. Portaria no 204, de 29 de janeiro de 2007. Regulamenta o financiamento e a transferência dos recursos federais para as ações e os serviços de saúde, na forma de blocos de financiamento, com o respectivo monitoramento e controle. Diário Oficial da União. Brasília, DF, 29 de jan. 2007.

Brasil (2013). Ministério da Saúde. Portaria $n^{\circ}$ 1554, de 30 de julho de 2013. Dispõe sobre as regras de financiamento e execução do Componente Especializado da Assistência Farmacêutica no âmbito do Sistema Único de Saúde (SUS). Diário Oficial da União. Brasília, DF, 30 de jul. 2013.

Brasil (2011). Ministério da Saúde. Lei n ${ }^{\circ}$ 12.401, de 28 de abril de 2011. Altera a Lei no 8.080, de 19 de setembro de 1990, para dispor sobre a assistência terapêutica e a incorporação de tecnologia em saúde no âmbito do Sistema Único de Saúde - SUS. Diário Oficial da União Brasília, DF, 28 de abr.2011.

Brasil (2011). Ministério da Saúde. Decreto n 7.646, de 21 de dezembro de 2011. Dispõe sobre a Comissão Nacional de Incorporação de Tecnologias no Sistema Único de Saúde e sobre o processo administrativo para incorporação, exclusão e alteração de tecnologias em saúde pelo Sistema Único de Saúde SUS, e dá outras providências. Brasília, DF, 21 de dez.2011.

Carvalho, D. M. (2019). Offset uma possível solução para problemática da judicialização dos medicamentos de alto custo no Brasil.

Catanheide, I. D., Lisboa, E. S., \& Souza, L. E. P. F. D. (2016). Characteristics of the judicialization of access to medicines in Brazil: a systematic review. Physis: Revista de Saúde Coletiva, 26(4), 1335-1356.

Costa, F. V., Da Motta, I. D., \& de Araújo, D. A. (2018). Judicialização da saúde: a dignidade da pessoa humana e a atuação do Supremo Tribunal Federal no caso dos medicamentos de alto custo. Revista Brasileira de Políticas Públicas, 7(3), 844-874. 
Research, Society and Development, v. 10, n. 2, e32610212700, 2021

(CC BY 4.0) | ISSN 2525-3409 | DOI: http://dx.doi.org/10.33448/rsd-v10i2.12700

Fonseca, J. Q. (2011) Acesso a medicamentos excepcionais na Bahia: o caso do Interferon Peguilado.

Lopes, F. D., \& de Campos Mello, T. R. (2018). A Judicialização e seu Papel na Efetivação do Direito à Saúde Pública. Revista de Gestão em Sistemas de Saúde, 7(3), 275-286.

Machado, M. A. D. Á., Acurcio, F. D. A., Brandão, C. M. R., Faleiros, D. R., Guerra Jr, A. A., Cherchiglia, M. L., \& Andrade, E. I. G. (2011). Judicialization of access to medicines in Minas Gerais state, Southeastern Brazil. Revista de Saúde Pública, 45(3), 590-598.

Magarinos-Torres, R., Pepe, V. L. E., Oliveira, M. A., \& Osorio-de-Castro, C. G. S. (2014). Medicamentos essenciais e processo de seleção em práticas de gestão da Assistência Farmacêutica em estados e municípios brasileiros. Ciência \& Saúde Coletiva, 19, 3859-3868.

Mendes, K.D.S., Silveira, R.C.C.P., Galvão, C.M. Revisão integrativa: método de pesquisa para incorporação de evidências na saúde e na enfermagem. Texto Contexto Enfer 2008;17(4):758-64.

Mendonça, S. M. F. (2016). Medicamentos de alto custo: a judicialização e o papel do Estado.

Oliveira et al.,2016. Perfil das demandas judiciais por medicamentos na Diretoria de Assistência Farmacêutica da Bahia no ano de 2015. In: Fórum Brasileiro Sobre Assistência Farmacêutica E Farmacoeconomia, 4, 2016. Salvador: jornal de assistência farmacêutica e farmacoeconomia, 2016. 1(1).

Portela, A. D. S., Leal, A. A. F., Werner, R. P. B., Simões, M. O. S., \& Medeiros, A. C. D. (2010). Políticas públicas de medicamentos: trajetória e desafios. Revista de Ciências Farmacêuticas Básica e Aplicada, 31(1).

Reis, A. R., \& de Oliveira, L. C. (2019). A Judicialização Dos Medicamentos De Alto Custo No Brasil E A Teoria Dos Custos Dos Direitos. Revista Digital Constituição e Garantia de Direitos, 12(2), 115-133.

Silva, L. C. D. (2013). Judicialização da saúde: em busca de uma contenção saudável. Âmbito Jurídico [Internet], 16(112), 1-7.

Souza, S.S, \& Costa, L..A; (2016). Implantação da Política de Assistência Farmacêutica do Estado. Jornal Assistência Farmacêutica e Farmacoeconomia, Bahia. 